\title{
The Immune System: A Variable Structure Control Perspective*
}

\author{
Anet J. N. Anelone* Harshal B. Oza* Sarah K. Spurgeon* \\ * School of Engineering and Digital Arts, University of Kent, \\ Canterbury, Kent CT2 7NT, UK (e-mail:ajna5@kent.ac.uk, \\ H.B.Oza@kent.ac.uk,S.K.Spurgeon@kent.ac.uk)
}

\begin{abstract}
A healthy immune system exhibits robustness to disease where the robust stability and performance depends on the immune response mechanism. This paper presents an analysis of the immune system from the viewpoint of variable structure control. The immune response function is regarded as a switch. The influence of candidate personal immune response functions inspired by both experimental and mathematical work from immunology on capturing this crucial dynamics is reviewed. Motivated by this analysis and knowledge of variable structure control, a discontinuous switch which yields more realism and an improvement in the stability and robustness of the immune system has been designed and studied. In particular, surfaces which are functions of immune cell concentrations are identified. These form analogous functions to the well-known sliding mode surfaces which are known to represent a special class of variable structure control. These functions can be seen from the biological viewpoint as system properties which the healthy immune system appears to drive to zero, a well-known characteristic of the sliding mode paradigm.
\end{abstract}

\section{INTRODUCTION}

The immune system is studied from a variable structure control perspective. Sliding mode control as described by Utkin (1977) is a special case of variable structure control and is a well-known robust control paradigm in control systems theory. The main features include inherent robustness and reduced order dynamics produced by allowing the system to evolve only on a user selected manifold in the state-space. In recent years, the immune system has been studied rigorously from a systems theoretical viewpoint as can be seen in Alexander and Wahl (2011); Iwami et al. (2009); Wing et al. (2006); Janeway et al. (2005) and the references therein. The results presented in this paper are motivated by the fact that the immune system can be regarded as a biological robust control system, with an inherent variable structure nature.

There is convincing evidence that characteristics of the immune system match those of other types of dynamical control system as can be seen from the work of DeBoer and Perelson (2013); Blyuss and Nicholson (2012); Alexander and Wahl (2011); Iwami et al. (2009, 2007); Utzny and Burroughs (2003). The study in Alexander and Wahl (2011); Bluestone et al. (2010); Wing et al. (2006); Janeway et al. (2005)) reveals that the adaptive immune system is a closed loop feedback which monitors antigen. The work in DeFranco et al. (2007); Wing et al. (2006) elucidates this sensing mechanism which is employed by the Antigen Presenting Cells along with naive T-cells. When the relevant signals are delivered, the control system is activated seeking to restore the system to its healthy antigenic steady-state. The control performance includes

\footnotetext{
ऋ This work was partially supported by EPSRC via research grant $\mathrm{EP} / \mathrm{J} 018295 / 1$.
}

suitable response time, good disturbance rejection, robustness to parameter variations and monitoring accuracy. The biological environment (cell population, reaction speed and biological activities) can be affected by several factors (natural evolution, aging, drugs, location) which can disturb the optimal operation of the immune system.

Biologists have regarded the immune system as a single structure which produces a linear response to the presence of antigen. The studies in Britton (2003); Brown and Rothery (1993) are examples of this. The population of specific attacking cells increases proportionally with the concentration of the specific antigen according to a constant defined by immunological experience of the cells. In control terms, it was considered analogous to a linear state regulator design with a fixed structure of the state feedback where the control parameter is set to meet performance goals.

Recent immunological experience and related mathematical models argue that an adaptive immune response comprises different structures involved with a potential switching mechanism as discussed in DeBoer and Perelson (2013); Blyuss and Nicholson (2012); Alexander and Wahl (2011); Iwami et al. (2009). The different structures correspond to different immunological states such as healthy, chronic autoimmune disease and recurrent infection. The adaptive immune response system switches from one subsystem to the other according to a switching logic. Biologically, it means that the states of the patient change according to the behaviour a switching logic modelled by the personal immune response function. Due to the sophisticated nature of the adaptive immune response, the position of the controller, the switching logic and the key parameters of each immunological structure are still the topic of intense research effort as can be seen in the con- 
tributions of DeBoer and Perelson (2013); Alexander and Wahl (2011); A.Toda and C.A.Piccirillo (2006); Miyara and Sakaguchi (2004).

Motivated by the apparent adaptive and variable structure nature of the immune system, it is argued in this paper that the natural biological design of the immune system fits into the philosophy of a variable structure system (VSS) defined in Utkin (1977). The study of the immune system is undertaken as a robust control system by introducing a purely discontinuous switch in the dynamics of the immune cells. The results show that this does not significantly change the overall dynamics. Furthermore, a detailed analysis of the resulting equilibria is also given that matches the qualitative behaviour reported in the literature. Motivated by the inherent robustness properties and the discontinuous nature of the immune cell activation dynamics, a new perspective involving expressions of attractor surfaces are established based on the principles of sliding mode control. The ensuing analysis shows mathematically that the naturally occurring robust immune system exhibits attraction to these surfaces in a similar way to that observed in controlled physical systems.

The novelty of this paper is the analysis of the mechanisms of the adaptive immune response of T-cells using variable structure control theory along with the proposition of a discontinuous function to model the inducement of the activation and proliferation of T-cells due to antigen stimulation.

The paper is organized as follows. Section 2 presents the model of the immune system. Section 3 presents the study of a new discontinuous immune response function and compares the overall qualitative behaviour obtained with other models appearing in the literature. Section 4 then identifies mathematically an attractive manifold and the analysis of convergence of the states of the system to this manifold. Section 5 presents some conclusions.

\section{ANALYSIS OF A SIMPLE MODEL OF THE IMMUNE RESPONSE}

The immune response is a defense mechanism responsible for protecting the body against entities judged to be harmful while causing minimum damage to the body itself. The immune system model considered in this paper is a third order model which describes the variation over time of the population of healthy target cells $T$, damaged cells (antigen concentration) $D$ and immune cells $C$ such as CD4+ or CD8+ T-cells. This representation is well established within the literature in the dynamics of disease as can be seen from Perelson and Nelson (1998); Smith and Leenheer (2003); Iwami et al. (2007). The population growth of healthy cells is assumed to be affine and target cells are produced at a rate $\lambda$. The production of antigen is induced by the damage and removal process of immune cells on target cells with an efficacy coefficient $\beta$. It is assumed that there are constant natural removal rates for each type of cell $(\mu, \alpha, \gamma)$, respectively. More details can be found in Iwami et al. (2007). The dynamical equations are given below

$$
\begin{aligned}
& \dot{T}=\lambda-\mu T-\beta T C \\
& \dot{D}=\beta T C-\alpha D \\
& \dot{C}=f_{P I}(D)-\gamma C
\end{aligned}
$$

The function $f_{P I}(D)$ is called the personal immune response function. It models the relationship between the concentration of antigen $(D)$ and the inducement of immune cells $(C)$. The triggering elements and the subsequent behaviour of the switches in the immune system are complex as described by Kim and Ahmed (2010); Kim et al. (2007). This paper looks at the personal immune response function as a switched control action because it changes the immunological states and it has a major impact on their dynamical behaviour and robustness.

\section{CANDIDATE PERSONAL IMMUNE RESPONSE FUNCTIONS}

Different mathematical representations of the personal immune response function have been studied in the literature and supported by theoretical or experimental immunological evidence as discussed by Iwami et al. (2007, 2009). The dynamical behaviour induced by different $f_{P I}(D)$ candidates corresponds to a range of clinical symptoms observed in autoimmune disease and described by Iwami et al. (2007); Alexander and Wahl (2011); Blyuss and Nicholson (2012). The way in which the total inducement of immune cells depends on the antigen density, $f_{P I}(D)$, can be represented by a linear, a Michaelis-Menten, a sigmoidal or a bell-shaped function. Iwami et al. (2007, 2009).

The linear function assumes that the production of immune cells is proportional to the concentration of antigen and is expressed as

$$
f_{P I}(D)=k D
$$

The average magnitude of activation/production of immune cells is encompassed by the parameter $k$. This linear candidate has been investigated in Iwami et al. (2007); Smith and Leenheer (2003). The system has two steadystates: one trivial equilibrium located at $T_{L 0}=\frac{\lambda}{\mu} ; D_{L 0}=$ $C_{L 0}=0$ corresponding to a healthy (tolerance) state and a non-trivial equilibrium located at $T_{L 1}=\frac{\alpha \gamma}{\beta k} ; D_{L 1}=\frac{\lambda}{\alpha}-$ $\frac{\mu \gamma}{\beta k} ; C_{L 1}=\frac{\lambda k}{\alpha \gamma}$ corresponding to an autoimmune disease state. The sign of the poles depends on the relation $\lambda \beta k-$ $\mu \gamma \gamma=0$. Therefore, the stability of the equilibria depends on the parameter values linked by the ratio $R_{0}=\frac{\beta k T_{L 0}}{\gamma \alpha}$. From Iwami et al. (2007) the trivial equilibrium is globally asymptotically stable (GAS) if $R_{0}<=1$ and the nontrivial equilibrium is GAS if $R_{0}>1$. The value of the parameter $k$ is varied whilst the other parameter values are non-negative constants. Since $\frac{d R_{0}}{d k}>0$, it has been shown that the stability of the healthy state shifts to the autoimmune state as $k$ increases. A small $k$ leads to a stable trivial equilibrium whilst a large $k$ makes the non trivial equilibrium stable.

The Michaelis-Menten function

$$
f_{P I}(D)=\frac{m D}{K_{m}+D}
$$


where $m$ is the maximum proliferation rate of immune cells and $K_{m}$ is the antigen concentration at which the proliferation rate is half maximal, has been investigated by Iwami et al. (2007); Brown and Rothery (1993). This nonlinear saturation function is an asymptotic switching curve. When $D \ll K_{m}, f_{P I}=\frac{m}{K_{m}} D$ so that the proliferation is directly proportional to the antigen concentration. When the antigen concentration is very large, $f_{P I}=m$ and the proliferation rate is saturated and independent of the antigen concentration. Although the MichaelisMenten function improves the realism by changing the expression of the two equilibria, the resultant qualitative behaviour of the system is similar to that of the linear approximation (2). Hence, the system with a linear or a Michaelis-Menten immune response function has stability and performance characteristics determined by parameters and their variation and the behaviour does not depend on initial conditions.

The influence of a Holling type III personal immune response function has been investigated in Iwami et al. (2007). This sigmoidal function is expressed as

$$
f_{P I}(D)=\frac{m D^{2}}{D^{2}+h^{2}}
$$

where $m$ is the maximum proliferation rate of immune cells and $h$ is the antigen concentration at which the proliferation rate is half maximal. It was demonstrated that a sigmoidal immune response function dramatically affects the qualitative behaviour of the dynamics. In fact, the system has three equilibria: a trivial equilibrium $E_{0}$ and two non-trivial equilibria $E_{-}$and $E_{+}$which exist if and only if $\lambda^{2} \beta^{2} m^{2}-4 \mu \gamma \alpha^{2} h^{2}(\mu \gamma+\beta m)>0$. The poles of the trivial equilibrium are $:-\mu ;-\alpha ;-\gamma$. Since biological parameters are positive, the poles are negative. Therefore, the system is always stable at the trivial equilibrium. It has been proven that whenever $E_{+}$exists it is always stable if $\mu>>\gamma$. $E_{-}$is a saddle equilibrium having a twodimensional stable manifold and a one dimensional unstable manifold. It was shown in Fig. 8 in Iwami et al. (2007) that $E_{-}$depicts a boundary surface between the absorbing domain of $E_{0}$ and $E_{+}$. When the antigen population is small the stable trivial equilibrium is reached whereas the stable non-trivial equilibrium is experienced when the antigen concentration is large. Thus, a sigmoidal switch modifies the dynamical behaviour and mathematical structures, leading to a bistable immune system. Hence, the immunological steady-states are robust against parameter variations and they depend on initial conditions, where the antigen concentration is particularly important.

Within the domain of engineering applications, a pure switch within the control strategy can yield interesting robustness properties. However, it is often not appropriate to implement a discontinuous control action in practical implementation. A solution to this problem is to use a step-like sigmoidal function for smoothing the discontinuity as discussed in Burton and Zinober (1986). It is interesting to note that the curvature of the biologically inspired sigmoidal function is different from the curvature of the typical sigmoidal switching function implemented in engineering applications. Indeed, the curvature of the Holling type III function is less steep than the curvature of the sigmoidal engineering switch. The "S" shape of this personal immune response function represents exponential

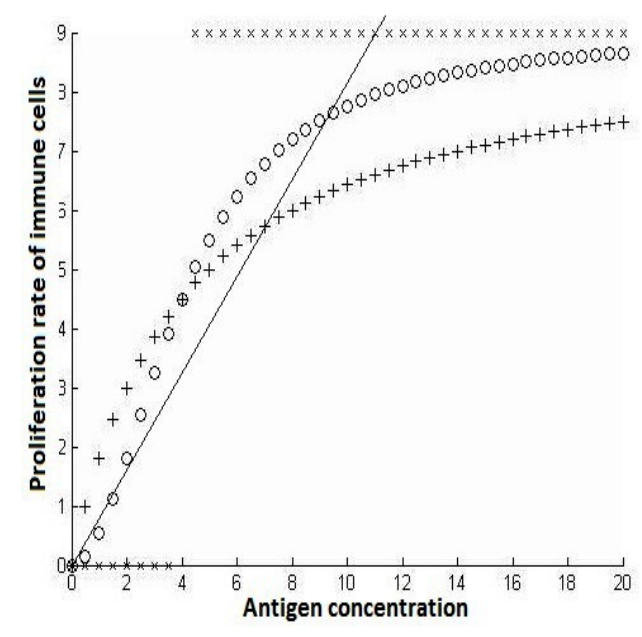

Fig. 1. Different personal immune response curves: - linear, + Michaelis-Menten, o sigmoidal and x discontinuous.

growth producing a rapid rate of change and incorporates saturation which decreases the rate of change as described by Britton (2003). Fundamentally in this sigmoidal representation, the nonlinearity is achieved by increasing the effect of $D$ and $K_{m}$ of the Michaelis Menton function. This function shows the tolerance of the switch when a low number of triggering elements are present which is an essential element of the biological system.

Table 1 summarises the influence of the candidate personal immune response function on the qualitative behaviour of the immune system. Figure 1 shows a comparison of the shapes of the different immune response switching curves. From this comparison, it can be deduced that the nature of $f_{P I}$ influences the stability, performance and robustness of the observed immunological states as well as their corresponding mathematical structures as in Iwami et al. (2007, 2009); Alexander and Wahl (2011).

\subsection{Discontinuous personal immune response proposed by control engineering perspective}

From a control engineering perceptive, this paper argues that the immune system operates as a variable structure control system because the activation and proliferation of

Table 1. Comparison of the mathematical structures and robustness of the immune system due to changes in the personal immune response function.

\begin{tabular}{|c|c|}
\hline $\begin{array}{l}\text { Personal immune } \\
\text { response function }\end{array}$ & $\begin{array}{l}\text { Qualitative } \\
\text { behaviour }\end{array}$ \\
\hline $\begin{array}{c}\text { Proportional to } \\
\text { antigen } \\
\text { concentration as in } \\
\text { (2), Smith and Leenheer }(2003)\end{array}$ & $\begin{array}{c}\text { Stability and } \\
\text { performance of } \\
\text { the two equilibria are } \\
\text { sensitive to parameter values }\end{array}$ \\
\hline $\begin{array}{c}\text { Michaelis Menten } \\
\text { as in (3), Iwami et al. (2007) }\end{array}$ & $\begin{array}{l}\text { Similar to } \\
\text { above }\end{array}$ \\
\hline $\begin{array}{c}\text { Sigmoidal } \\
(\text { Holling type III) } \\
(4), \text { Iwami et al. (2007) }\end{array}$ & $\begin{array}{c}\text { Bistability } \\
\text { Saddle equilibrium } \\
\text { Robust performance }\end{array}$ \\
\hline $\begin{array}{c}\text { Discontinuous switch } \\
\text { based on antigen population } \\
(5)\end{array}$ & $\begin{array}{c}\text { Bistability } \\
\text { Unstable equilibrium } \\
\text { Robust performance }\end{array}$ \\
\hline
\end{tabular}


T-cells switches immunological states depending on the antigen density uptake.

Full consideration of the characteristics of the model used for the biological switch is interesting because it illustrates the synergies between the immune system response, as a closed-loop control system, and the behaviour of dynamical systems represented by ODEs where a discontinuous feedback control is applied. Moreover, it contributes to new understanding and/or reinforcement of existing biological knowledge of the underlying phenomena. The immune system itself is a robust and highly sophisticated biological system and thus including discontinuous behaviour of the switch models the robustness properties. In this paper, a discontinuous personal immune response has been developed. Mathematically, the proposed discontinuous personal immune response function is given as:

$$
f_{P I}(D)=m \frac{1}{2}\left(\frac{D-h}{\|D-h\|}+1\right)
$$

A sign function based on $D$ is effectively used to produce an on/off immunological switch. The sign function is shifted in the positive quadrant so that its output is always positive and biologically meaningful. To encompass the tolerance characteristics of the immune system, an antigen threshold $h$ is set to match with the concentration of damaged cells at the point where the proliferation rate is half of the maximum for a sigmoidal response used in Iwami et al. (2007). The immune response is induced when $D$, the concentration of antigen, exceeds that threshold. The amplitude of the response $m$, is the saturation (maximum) proliferation rate of the attacking immune cells. Unlike the personal immune response function currently used in the literature, the discontinuous $f_{P I}(D)$ is not inspired from biology. It does not aim to be the "ideal" personal immune response function. It has a step like behaviour made up of a brutal (sharp) change in its output, the proliferation of attacking immune cells. The discontinuous behaviour also yields antigen tolerance but implies an instantaneous change of behaviour (activation/inhibition). The behaviour of the discontinuous personal immune response function is the switching logic which changes the mathematical structure of the immune system whilst moving between the healthy and the autoimmune diseased state.

The steady-state analysis performed in Iwami et al. (2007) is carried out for (5). A trivial equilibrium is found analytically at $T_{0}=\frac{\lambda}{\mu}, D_{0}=0, C_{0}=0$. The Jacobian is evaluated at this point. The roots of the characteristic equation are $:-\mu ;-\alpha ;-\gamma$. Since death rates are always positive, the poles are always negative. Therefore, this equilibrium is always stable. This trivial equilibrium depicts the case where there are no symptoms of autoimmune disease. Therefore, it represents the healthy (tolerance) state of the immune system. It should be noted that the discontinuous element of $f_{p i}(D)$ in equation (5) influences the analytical expression of the equilibrium points of the system (1). As a result, the expression for the equilibrium points depends on the output of the personal immune response function, especially on the output of $\operatorname{sign}(D-h)$.

Considering the case where $S=-1=>f_{P I}=0$ which means that the population of antigen is less than the threshold, the (trivial) equilibrium point is obtained. The second outcome is reached when the concentration of antigen is equal to the antigen threshold. Mathematically, $S=0<=>f_{P I}=m / 2$ i.e. the immune response is in a transient phase so it produces half the maximum proliferation rate of attacking immune cells. The transient equilibrium point is:

$$
T_{1}=\frac{2 \lambda \gamma}{\beta m+2 \mu \gamma} ; D_{1}=\frac{\beta \lambda m}{\alpha(\beta m+2 \mu \gamma)} ; C_{1}=\frac{m}{2 \gamma}
$$

The Jacobian at this equilibrium point is evaluated. The characteristic equation is a third order polynomial having positive coefficients for all powers of $s$. The Routh-Hurwitz stability criteria are applied. The system is unstable at the transient equilibrium (6). Hence, in the current immunological scenario, the immune response is activated and it is in an unstable transient phase. Now, the immunological scenario when $D>h<=>S=1<=>f_{P I}=m$, the antigen threshold is surpassed and the personal immune response function delivers the maximum proliferation rate of immune cells. the resultant equilibrium point is expressed as:

$$
T_{2}=\frac{\lambda}{\mu+\left(\frac{m \beta}{\gamma}\right)} ; D_{2}=\frac{m \beta \lambda}{m \beta \alpha+\alpha \mu \gamma} ; C_{2}=\frac{m}{v}
$$

This non-trivial equilibrium (7) is associated with an active immune response for chronic auto immune disease. The Jacobian at this equilibrium $(7)$ is evaluated and its eigenvalues are: $-\alpha ;-\gamma ;-\left(\mu+\left(\frac{m \beta}{\gamma}\right)\right.$. Since biological parameters are always positive, the eigenvalues are all negative. Therefore, the system (1) is always stable at the equilibrium point (7). Hence, when the concentration of antigen is above the threshold, the immune response is activated and a stable chronic autoimmune disease state is exhibited by the patient.

Simulations are conducted with parameter values in (4) taken from Iwami et al. (2007). Numerically, the healthy equilibrium is at $\left(T_{0}=100 ; D_{0}=C_{0}=0\right)$, the transient equilibrium $\left(T_{1}=97.518 ; D_{1}=0.2256 ; C_{1}=0.0051\right)$ and the autoimmune disease equilibrium $\left(T_{2}=2.70357 ; D_{2}=\right.$ $\left.8.8451 ; C_{2}=7.197\right)$. The autoimmune disease equilibrium exists when $h<D_{2} \cdot h=D_{-}=0.2256$ which is the value of the saddle antigen equilibrium when a sigmoidal switch is applied. This selection facilitates the comparison of the dynamical behaviour by remaining in the same numerical range as in Iwami et al. (2007). Considering the simulation with initial conditions $T(0)=100 ; D(0)=0.2 ; C(0)=0$, the population of target cells remains large and the other cell populations are driven to zero. The resultant healthy state of the system does not change since the antigen concentration is less than the threshold. This behaviour has been suggested and demonstrated by previous theoretical and experimental publications reported in Wing et al. (2006); Iwami et al. (2007); Alexander and Wahl (2011); Blyuss and Nicholson (2012).

When the antigen concentration is above the threshold, the trajectories of the different cell types move first towards the unstable equilibrium because it incorporates an attractive manifold. This behaviour is not shown on Figure 2 due to scale because the mechanism of dormancy is faster. 


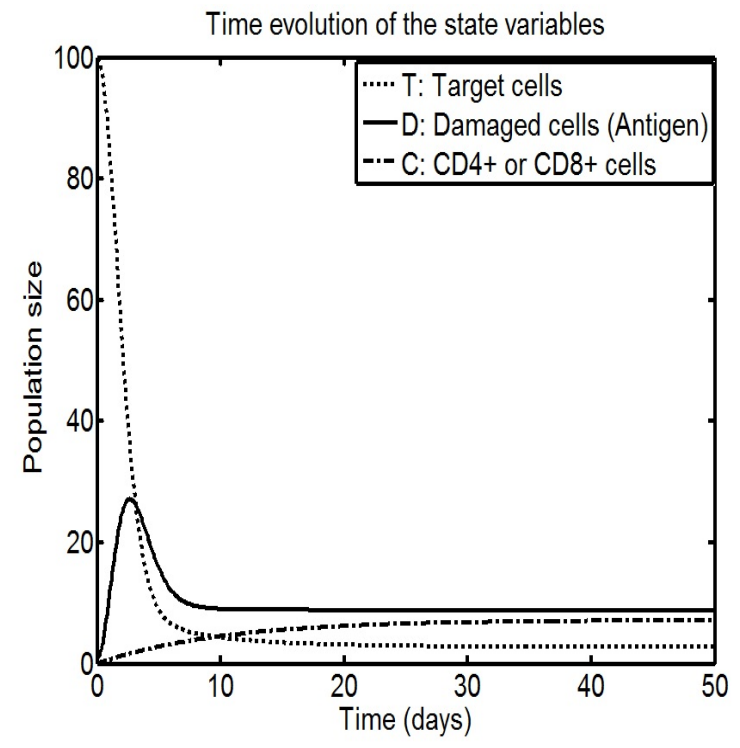

Fig. 2. Simulation of the state of autoimmune disease with initial conditions: $\mathrm{T}(0)=100 ; \mathrm{D}(0)=1 ; \mathrm{C}(0)=0$.

Indeed, the response time of the immune system with a discontinuous switch is much smaller than that of the sigmoidal switch studied in Fig. 5 in Iwami et al. (2007).

The response time in Figure 2 is very rapid with the population of target cells rising whilst the population of target cells decreases. The number of healthy cells becomes negligible compared to the number of immune cells which stabilise at a high level. At the same time the antigen concentration reaches a peak value and then decreases and remains constant. This dynamical behaviour which models acute and chronic symptoms is typical of the disease dynamics shown in Iwami et al. (2007); Smith and Leenheer (2003). This antigen peak is caused by the coupling between target cells and immune cells. The transition from the trivial equilibrium to the nontrivial equilibrium makes the product of the target cell and immune cell population reach its maximum value for producing antigen. The non-trivial autoimmune disease equilibrium is as attractive as the healthy equilibrium. The simulations confirm the analytical results. The qualitative behaviour of the system is affected by the choice of initial conditions.

\section{ATTRACTIVE SLIDING SURFACE}

This section identifies certain attractive surfaces in the state-space of dynamics (1) to build engineering applications evidence of robustness in the immune system in a way generally followed in applications of variable structure systems Utkin (1977).

Consider model (1). Consider the following equation of

$$
s=D+C .
$$

Sliding mode control synthesis typically begins with such a surface so that the closed-loop system exhibits desired behaviour when constrained to this surface. The first design guideline is thus to select an appropriate surface underpinning a stable behaviour.

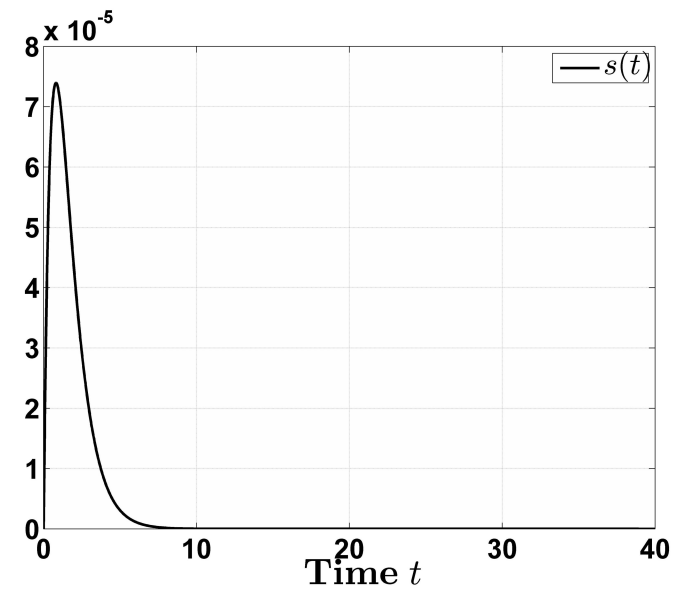

Fig. 3. Evolution of the switching surface $s(t)$

In the present case, there is no synthesis being carried out. Rather, motivated by understanding the naturally occurring sliding surfaces, the above choice of sliding line is motivated by the fact that a stable healthy immune system would drive the antigen concentration $D$ and the immune cell population $C$ to zero. Motivated by the usual guidelines of sliding mode control, it is then of interest to see if the temporal derivative of sliding line (8) vanishes along the system trajectories (1). The temporal derivative of the surface (8) can be obtained as follows:

$$
\dot{s}=\dot{D}+\dot{C}=\beta T C-\alpha D+f_{P I}(D)-\gamma C
$$

Considering the case of a healthy immune system where $f_{P I}(D)=0$, we have the following simplification,

$$
\dot{s}=\beta T C-\alpha D-\gamma C
$$

It should be noted that $s>0$ for all $D, C$ as these form concentrations of cell population. Hence an expression similar to the well-known reachability condition as described in Utkin (1977) can be obtained as follows:

$$
s \dot{s} \leq|s|(\beta T C-\alpha D-\gamma C)
$$

Following the discussion in Section 2, it should be noted that unlike the traditional sliding mode control, the function $f_{P I}$ does not switch around the sliding surface $s$. Rather the asymptotic attraction to or repellency for this surface $s$ is obtained due to a certain value of $f_{P I}$. The surface $s$ is rendered attractive by a healthy immune system if the following expression holds true:

$$
\beta T C-\alpha D-\gamma C<0
$$

Fig. 3 shows the time history of the surface $s(t)$ and Fig. 4 shows the time history of the function $\beta T C-\alpha D-\gamma C$. It can be seen that the function $\beta T C-\alpha D-\gamma C$ always remains negative thereby rendering $s=0$ the domain of attraction. It can also be claimed that those values of the model constants $\alpha, \beta, \gamma$ that induce such a behaviour forms a sufficient condition for $s=0$ to be attractive, thereby inducing a stable healthy steady-state.

Although, there are similarities in the above analysis to variable structure control, there are some dissimilarities, too. Sliding mode systems typically have uniform decay of 


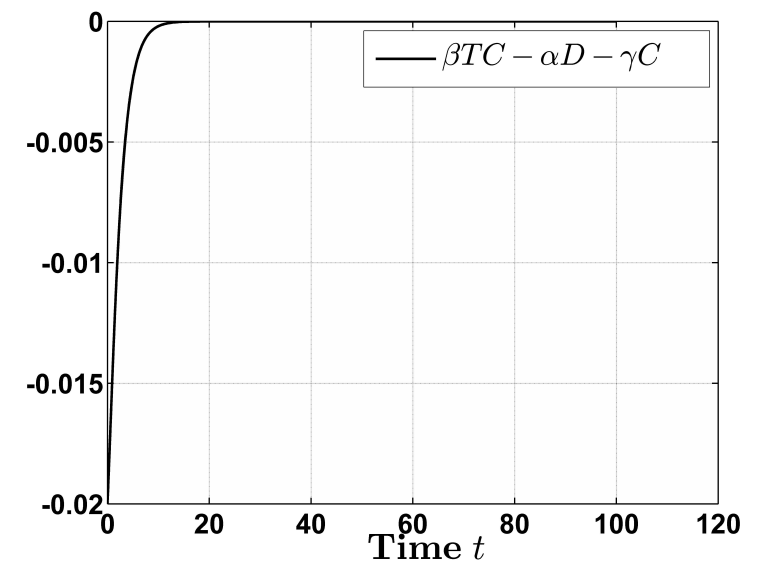

Fig. 4. Function $\beta T C-\alpha D-\gamma C$

$s(t)$ to zero. An exponential decay is always preferable in control of physical systems. Since there is no synthesis in the present case, there is no control over how $s(t)$ decays. The resulting non-uniform decay of the surface $s(t)$ and the initial increase in the value of $s(t)$ are distinctly different behaviours when compared to those prescribed by a typical variable structure control. However, from the biological view-point, such behaviour is not new as can be seen from Fig. 5 and 8 of Iwami et al. (2007). The reason for this is that the two stable steady-states are separated by an unstable or saddle transient equilibrium. The immune system typically requires a certain threshold of a particular cell population to be surpassed before it becomes active. The plot in Fig. 3 exhibits such behaviour where, after a certain point in time (between two and three units), the immune system becomes active and drives both the populations $D$ and $C$ to zero. Hence, the attractive surface $s$ thus identified can be seen as a valid candidate model for capturing key dynamics of the natural healthy immune response.

\section{CONCLUSION}

The effects on the immune system model of different candidate personal immune response functions inspired from biology have been analysed from a control engineering perspective. It is deduced through analytical and numerical evidence that the personal immune response function can be regarded as a discontinuous switching function because it changes the states according to the antigen concentration. Thus the immune system has been studied as a variable structure control system. Subsequently, an appropriate expression of a naturally occurring attractor surface was identified. The resulting immune system satisfies established conditions to render the sliding surface attractive. Consequently, the similarities and differences in behaviour between a discontinuous model of the immune response and a classical model of a discontinuous feedback control are highlighted.

\section{ACKNOWLEDGEMENTS}

The authors would like to thank Dr. Najl V. Valeyev of University of Exeter, UK for his comments and interactions in improving the paper.

\section{REFERENCES}

Alexander, H.K. and Wahl, L.M. (2011). Self-tolerance and autoimmunity in a regulatory t cell model. Bulletin of Mathematical Biology, 73(1), 33-71.

A.Toda and C.A.Piccirillo (2006). Development and function of naturally occurring $\mathrm{cd} 4+\mathrm{cd} 25+$ regulatory $\mathrm{t}$ cells. J. Leukoc. Biol., 80(1), 458-470.

Bluestone, J., K.Herold, and Eisenbarth, G. (2010). Genetics, pathogenesisandclinical interventions in type 1 diabetes. Nature, 464, 1293-1300.

Blyuss, K. and Nicholson, L. (2012). The role of tunable activation thresholds in the dynamics of autoimmunity. J. Theor. Bio., 308, 45-55.

Britton, N.F. (2003). Essentials of mathematical biology. Berlin: Spinger.

Brown, D. and Rothery, P. (1993). Models in biology: Mathematics, Statistics and computing. John Wiley \& Sons.

Burton, J.A. and Zinober, A.S.I. (1986). Continuous approximation of variable structure control. International Journal of Systems Science, 17(6), 875-885.

DeBoer, R. and Perelson, A. (2013). Quantifyingtlymphocyteturnover. J.Theor.Biol.

DeFranco, A., Locksley, R., and Robertson, M. (2007). Immunity: The immune response in infectious and inflammatory disease. New Science Press Ltd.

Iwami, S., Takeuchi, Y., Miura, Y., Sasaki, T., and Kajiwara, T. (2007). Dynamical properties of autoimmune disease models: tolerance, flare-up, dormancy. J. of Theoretical Biology, 246, 646-659.

Iwami, S., Y.Takeuchi, K.Iwamoto, Y.Naruo, and Yasukawa, M. (2009). A mathematical designof vector vaccine against autoimmune disease. J.Theor.Biol., 256, $382-392$.

Janeway, C., Travers, P., Walport, M., and Shlomchik, M. (2005). Immunobiology: The Immune System in Health and Disease. Garland, New York, 6th edition.

Kim, P. and Ahmed, R. (2010). Features of responding t cells in cancer and chronic infection. Current Opinion in Immunology, 22(2), 223-230.

Kim, P., P.P.Lee, and Levy, D. (2007). Modeling regulation mechanisms in the immune system. J. Theor. Biol., 246, 33-69.

Miyara, M. and Sakaguchi, S. (2004). Natural regulatory t cells: mechanisms of suppression. TRENDS Mol. Med., 132(1), 108-116.

Perelson, A.S. and Nelson, P.W. (1998). Mathematical analysis of HIV-1 dynamics in Vivo. SIAM Review, 41(1), 3-44.

Smith, H. and Leenheer, P.D. (2003). Virus Dynamics: A Global Analysis. SIAM J. Appl. Math, 63(4), 1313-1327.

Utkin, V. (1977). Variable structure systems with sliding modes. IEEE Transactions on Automatic Control, $22(2), 212-222$.

Utzny, C. and Burroughs, N.J. (2003). Pertubation theory analysis of competition in a heterogenous population. Physica D, 175, 109-126.

Wing, K., Fehervari, Z., and Sakaguchi, S. (2006). Emerging possibilities in the development and function of regulatory t cells. Int. Immunol., 18(1), 991-1000. 